\title{
A no-scale supergravity framework for sub-Planckian physics
}

\author{
John Ellis, ${ }^{1, *}$ Dimitri V. Nanopoulos, ${ }^{2, \dagger}$ and Keith A. Olive ${ }^{3, \neq}$ \\ ${ }^{1}$ Department of Physics, Theoretical Particle Physics and Cosmology Group, King's College London, \\ London WC2R 2LS, United Kingdom and Theory Division, CERN, CH-1211 Geneva 23, Switzerland \\ ${ }^{2}$ George P. and Cynthia W. Mitchell Institute for Fundamental Physics and Astronomy, \\ Texas A\&M University, College Station, Texas 77843, USA; Astroparticle Physics Group, \\ Houston Advanced Research Center (HARC), Mitchell Campus, Woodlands, \\ Texas 77381, USA; and Division of Natural Sciences, Academy of Athens, \\ 28 Panepistimiou Avenue, Athens 10679, Greece \\ ${ }^{3}$ William I. Fine Theoretical Physics Institute, School of Physics and Astronomy, \\ University of Minnesota, Minneapolis, Minnesota 55455, USA
}

(Received 28 October 2013; published 3 February 2014)

\begin{abstract}
We propose a minimal model framework for physics below the Planck scale with the following features: (i) it is based on no-scale supergravity, as favored in many string compactifications, (ii) it incorporates Starobinsky-like inflation, and hence is compatible with constraints from the Planck satellite, (iii) the inflaton may be identified with a singlet field in a seesaw model for neutrino masses, providing an efficient scenario for reheating and leptogenesis, (iv) supersymmetry breaking occurs with an arbitrary scale and a cosmological constant that vanishes before radiative corrections, and (v) regions of the model parameter space are compatible with all LHC, Higgs boson, and dark matter constraints.
\end{abstract}

DOI: $10.1103 /$ PhysRevD.89.043502

PACS numbers: $98.80 . \mathrm{Cq}, 04.65 .+\mathrm{e}, 12.60 . \mathrm{Jv}$

There are two extensions of the Standard Model of particle physics that seem particularly well motivated: heavy singlet (right-handed) neutrinos to generate mixing and small masses for the standard left-handed neutrinos via the seesaw mechanism [1]; and supersymmetry [2] to stabilize the electroweak scale, facilitate grand unification, etc. Likewise, there are three well-motivated extensions of the standard big bang model of cosmology-baryogenesis [3], dark matter [4], and inflation [5] - to explain the largescale homogeneity of the universe and the fluctuations in the cosmic microwave background (CMB). There are also popular connections between these extensions of the Standard Model: for example, $C P$ violation in the decays of the heavy singlet (right-handed) neutrinos might have generated a lepton asymmetry that would subsequently have been converted into a baryon asymmetry via sphaleron interactions [6], supersymmetry provides a natural dark matter candidate [7,8], and the inflaton might have been a heavy singlet sneutrino [9].

The purpose of this paper is to propose a minimal framework that combines all of these desirable features. Low-energy particle physics is described by the minimal $\mathcal{N}=1$ supersymmetric extension of the Standard Model (MSSM), which is supplemented by a supersymmetric type 1 seesaw heavy neutrino sector. One of the heavy sneutrinos is identified as the inflaton [9], and its decays generate a lepton (and hence a baryon) asymmetry [6].

\footnotetext{
*John.Ellis@cern.ch

dimitri@physics.tamu.edu

\$olive@physics.umn.edu
}

The inflaton is embedded in a no-scale supergravity sector [10] together with another singlet field [11-13], in a form that could emerge naturally as the low-energy effective field theory obtained from some string compactification [14]. This form yields an effective potential and hence a spectrum of perturbations identical to the Starobinsky $R+R^{2}$ model [15], which is comfortably compatible with data from the Planck satellite [16]. We display a choice of superpotential for these fields that generates supersymmetry breaking with an arbitrary magnitude and zero vacuum energy at the tree level. We show that there are regions of the model parameter space that are compatible with the measured mass of the Higgs boson, supersymmetric dark matter, and the absence of a supersymmetric signal at the LHC.

Because we incorporate supersymmetry and consider cosmology, our model is formulated within an $\mathcal{N}=1$ supergravity framework [17]. This is characterized by a Hermitian Kähler potential $K\left(\phi_{i}, \phi_{j}^{*}\right)$ and a holomorphic superpotential $W\left(\phi_{i}\right)$, where the $\phi_{i}$ are complex scalar fields in chiral supermultiplets, in the combination $G \equiv K+\ln W+\ln W^{*}$. In order to have an effective potential that vanishes over a continuous range of parameters in the presence of supersymmetry breaking, and that is motivated by generic string compactifications [14], we choose a no-scale form for $K$ that has an underlying $\mathrm{SU}(N, 1) / \mathrm{SU}(N) \times \mathrm{U}(1)$ symmetry [18]. For the purposes of our discussion here, we display an example with $N=2$ that can be extended to include, e.g., the fields of the MSSM. In this case there are two equivalent field representations that are frequently used: 


$$
K=-3 \ln \left(T+T^{*}-|\phi|^{2} / 3+\cdots\right)
$$

and

$$
K=-3 \ln \left(1-\left|y_{1}\right|^{2} / 3-\left|y_{2}\right|^{2} / 3-\cdots\right) .
$$

In the first formulation, the $T$ field may represent a string modulus and the $\phi$ a generic matter field, whereas in the second formulation both $y_{1,2}$ may be matter fields.

It was shown in $[11,12]$ that the $N=2$ no-scale model can yield an effective potential identical to that of the Starobinsky $R+R^{2}$ model for various suitable choices of the superpotential $W[19]$. Here, we discuss one example that was introduced in [11] and explored in more detail in [12], namely,

$$
W=M\left[\frac{y_{1}^{2}}{2}\left(1+\frac{y_{2}}{\sqrt{3}}\right)-\frac{y_{1}^{3}}{3 \sqrt{3}}\right] .
$$

Unless explicitly denoted, we will work in Planck units $M_{P}^{2}=1$, where $M_{P}^{-2}=8 \pi G_{N}$ refers to the normalized Planck mass. Equation (3) represents the minimal WessZumino (WZ) model [2] for the no-scale field $y_{1}$, with a minimal interaction $y_{1}^{2} y_{2}$ with the other no-scale field $y_{2}$. Note that the modular factor $\left(1+y_{2} / \sqrt{3}\right)$ arises from the field redefinition from the $(T, \phi)$ basis. In general, the modular weight of a superpotential term of a field dimension $D$ is $3-D$.

The model described by Eq. (3) reproduces the effective potential of the Starobinsky model for $\mathcal{R} e y_{1}$ when a suitable stabilizing term $\propto y_{2}^{4}$ is introduced into the noscale Kähler potential [12,20,21]

$$
K=-3 \ln \left(1-\frac{\left|y_{1}\right|^{2}+\left|y_{2}\right|^{2}}{3}+\frac{\left|y_{2}\right|^{4}}{\Lambda^{2}}\right),
$$

with $\Lambda \lesssim 0.1 M_{P}$, as discussed in [12].

The no-scale structure explored here is subject to radiative corrections, which depend on the ultraviolet completion of the theory and are thought to play an essential role in determining the scale of supersymmetry breaking [22]. Since a discussion of the ultraviolet completion lies beyond the scope of this paper, which is concerned with sub-Planckian physics, and since the scale of inflation is much higher than the supersymmetrybreaking scale, we leave a more complete discussion of the structure of these radiative corrections for future work.

Having discussed how inflation may be embedded in a no-scale framework typical of a generic string compactification, we now discuss how it may be connected with lower-energy physics. The first issue is the possible identification of the inflaton field $y_{1}$ with some field appearing in (a motivated extension of) the Standard Model.
The forms of the interactions in (3) require $y_{1}$ to be a gauge-singlet field, and the minimal possibility is to identify it with one of the singlet (right-handed) neutrinos in the minimal type 1 seesaw extension of the Standard Model (a singlet sneutrino). Thus, we can add a Yukawalike term to the superpotential of the Standard Model,

$$
W_{Y}=\lambda H_{2}^{i} L_{i} y_{1}
$$

where $\mathrm{H}_{2}$ is the same Higgs doublet giving mass to the uplike quarks and $L$ is a left-handed lepton doublet. The index $i$ is an SU(2) index. In principle, we expect three righthanded singlet neutrinos, but we associate only one (or one linear combination of the three) with the inflaton. Remarkably, the order of magnitude of the mass parameter $M$ in (3) required to yield the right size of primordial scalar perturbations, namely, $\sim 10^{13} \mathrm{GeV}$, lies within the range favored in such seesaw models of neutrino masses and mixing. Furthermore, previous studies have shown that such a sneutrino inflationary scenario can comfortably generate a lepton asymmetry of the size required for successful leptogenesis $[9,23]$.

However, there is one issue that needs to be addressed, namely, that of lepton-number violation. When $y_{1}$ is identified as a sneutrino, in addition to the Majorana mass-generating $\Delta L=2$ interaction that conserves $R$ parity, there is a trilinear $\Delta L=3$ interaction that violates $R$ parity, implying that the LSP is, in principle, unstable. However, its lifetime would be very long [24]. If we identify the lightest supersymmetric particle (LSP) with the lightest neutralino $\chi$, on the basis of diagrams such as that in Fig. 1, we estimate that its lifetime is

$$
\tau_{\mathrm{LSP}} \sim \frac{192 \pi^{3} M_{P}^{2}(M \tilde{m})^{4}}{g^{2} \lambda^{6} m_{\chi}^{5} v^{6}},
$$

where $v$ is the Higgs vacuum expectation value (vev), $\tilde{m}$ is a typical supersymmetry-breaking soft mass, $\lambda$ is the Yukawa coupling between the light doublet and heavy singlet neutrinos, and $g$ is a gauge coupling. Taking $M \sim 10^{13} \mathrm{GeV}, \tilde{m} \sim 1 \mathrm{TeV}$ and $\lambda=\mathcal{O}(1)$, this estimate

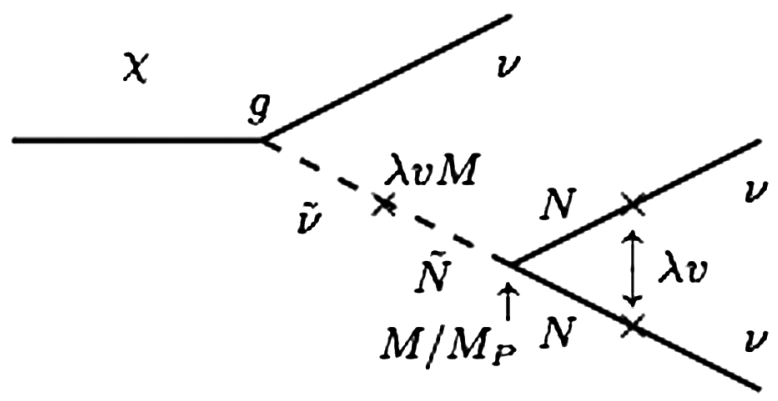

FIG. 1. Diagram for $\chi \rightarrow 3 \nu$ decay. The $\times$ symbols denote light $\nu$-heavy $N$ mixing via a Dirac Yukawa coupling as expected in a type 1 seesaw model. 
yields a LSP lifetime exceeding $10^{40}$ years. For smaller $\lambda$, as required to obtain a suitable reheat temperature, the lifetime of the neutralino will be substantially longer.

As the inflaton is directly coupled to Standard Model fields through (5), reheating and leptogenesis can proceed through the out-of-equilibrium decay of the heavy singlets [6]. The decay rate of $y_{1}$ can be approximated as $\Gamma_{D} \simeq \lambda^{2} M /(16 \pi)$, which is smaller than the Hubble rate at the end of inflation, $H_{I} \simeq M / 2$. The (instantaneous) reheating temperature $T_{R}$ is easily estimated to be

$$
T_{R} \simeq\left(\frac{5}{\pi^{3} N}\right)^{1 / 4}\left(\frac{\lambda^{2} M M_{P}}{\sqrt{32 \pi}}\right)^{1 / 2} \sim 2 \times 10^{14} \lambda \mathrm{GeV},
$$

assuming decay at $\Gamma_{D} \simeq \frac{3}{2} H$, where $N$ is the number of degrees of freedom at the time of decay, which we take to be the MSSM value of $885 / 4$.

High reheat temperatures are known to give rise to cosmological problems associated with gravitino production. Although gravitinos never dominate the energy density of the Universe if $m_{3 / 2} \sim 10 \mathrm{TeV}$, gravitino decay to neutralinos, $\chi$, can lead to an overabundance of the lightest supersymmetric particle that provides cold dark matter [8]. The abundance of gravitinos can be determined in terms of the reheat temperature as $[8,25]$

$$
\frac{n_{3 / 2}}{s} \simeq 2.4 \times 10^{-12}\left(\frac{T_{R}}{10^{10} \mathrm{GeV}}\right),
$$

where $s$ is the entropy density, assuming that the gravitino is much heavier than the gluino. In order to satisfy the upper limit on the abundance of neutralinos, $\Omega_{\chi} h^{2}<0.12$, we must ensure that [26]

$$
\frac{n_{3 / 2}}{s} \lesssim 4.4 \times 10^{-13}\left(\frac{1 \mathrm{TeV}}{m_{\chi}}\right),
$$

which leads to an upper limit on the Yukawa coupling $\lambda$ :

$$
\lambda \lesssim 10^{-5} .
$$

This result implies that one of the neutrino Yukawa couplings may be comparable to the electron Yukawa coupling and suggests that the mass of the lightest neutrino generated by the seesaw mechanism should be of the order $10^{-10}-10^{-9} \mathrm{eV}$. We note that the small value of $\lambda$ assures out-of-equilibrium decay and hence the possibility of successful leptogenesis.

Sneutrino inflation is not the only possibility within our no-scale framework. In some extensions of the Standard Model with extended gauge groups the heavy neutrinos are not singlets, but there may be other gauge-singlet superfields. One such example is the minimal flipped SU(5) grand unified theory (GUT) [27], where the heavy neutrinos are embedded in $\mathbf{1 0}$ representations $F_{i}$, and the inflaton may be identified with one of the other gauge- singlet fields $\phi_{i}$ in the model, which are expected to have trilinear couplings. The relevant couplings are of the form $\lambda_{6 i j} F_{i} \bar{H} \phi_{j}+\lambda_{8 i j k} \phi_{i} \phi_{j} \phi_{k}$, where $\bar{H}$ denotes a $\overline{10}$ Higgs representation with a GUT-scale vev, $V$, and one of the $\phi$ has a vev of similar magnitude. In this case, there is mixing between the heavy neutrinos and the singlet fields [28]; the lightest of their mass eigenstates may very well have a mass of $\sim 10^{13} \mathrm{GeV}$, as required for the inflaton, and it would, in general, have a trilinear self-coupling, as required (3) in our no-scale Starobinsky inflationary scenario. Previous studies have shown that this model can also lead to successful leptogenesis [28], but we do not enter here into details of the heavy sneutrino or singlet sector.

We now address another issue concerning lower-energy physics, namely, supersymmetry breaking. One of the attractive features of the original no-scale model [18] was that it accommodated local supersymmetry breaking in the form of an arbitrary gravitino mass $m_{3 / 2}=e^{G / 2}$ with zero vacuum energy (cosmological constant) before the calculation of quantum corrections. This even suggested a perturbative mechanism for the dynamical determination of $m_{3 / 2}$ [22]. Supersymmetry breaking can be accommodated within our framework by simply adding a constant (modular weight 3) term to the superpotential [(3)],

$$
W_{S S B}=r\left(1+\frac{y_{2}}{\sqrt{3}}\right)^{3} \text {. }
$$

It is easy to check that, as $\Lambda \rightarrow \infty$ in the Kähler potential (4), the parameter $r$ does not appear in the effective potential, implying that this is a further generalization of the no-scale Starobinsky models discussed previously in $[11,12]$. When $\Lambda$ is finite, the effective potential does depend on $r$, but this dependence drops out when $y_{2}=0$, as we enforce by requiring $\Lambda \lesssim 0.1 M_{P}$ [12]. It is also easy to check the magnitude of local supersymmetry breaking $m_{3 / 2}=r$ while the vacuum energy vanishes, as in the original no-scale model [18]. In (11) we treated $r$ as a parameter to be derived (presumably) from string dynamics. It would be interesting to resurrect the possibility of determining $r=m_{3 / 2}$ dynamically via quantum corrections in the low-energy effective theory, but we do not discuss that possibility here. Note that, in the presence of both nonvanishing $r$ and finite $\Lambda$, the modulus $y_{2}$ is strongly stabilized with a mass (for both real components) $m_{y_{2}}=6 \sqrt{2} r / \Lambda=6 \sqrt{2} m_{3 / 2} M_{P} / \Lambda \gg m_{3 / 2}$.

Next, we consider the extension of the model $(3,4,11)$ to Standard Model particles and their supersymmetric partners $y_{\mathrm{SM}}$. In the spirit of the no-scale approach, we embed them within the logarithm in the Kähler potential:

$K \ni-3 \ln \left(1-\left|y_{1}\right|^{2} / 3-\left|y_{2}\right|^{2} / 3-\left|y_{\mathrm{SM}}\right|^{2} / 3-\cdots\right)$.

We assume that the superpotential for the Standard Model superfields has the form 


$$
W_{\mathrm{SM}}=W_{2}\left(y_{\mathrm{SM}}\right)\left(1+\frac{y_{2}}{\sqrt{3}}\right)^{\beta}+W_{3}\left(y_{\mathrm{SM}}\right)\left(1+\frac{y_{2}}{\sqrt{3}}\right)^{\alpha} \text {, }
$$

where $W_{2,3}\left(y_{\mathrm{SM}}\right)$ are bilinear and trilinear in the Standard Model superfields, respectively, in which case the soft supersymmetry-breaking mass-squared bilinear and trilinear parameters are

$$
m_{0}=0, \quad B_{0}=(\beta-1) m_{3 / 2}, \quad A_{0}=\alpha m_{3 / 2} .
$$

The choice of modular weights $\alpha=0, \beta=1$ for the trilinear and bilinear terms, respectively, corresponds to the pure no-scale option $m_{0}=B_{0}=A_{0}=0$, in which case the only possibility for introducing supersymmetry breaking into the low-energy sector is via a nonminimal gauge kinetic term, generating the nonzero gaugino masses $m_{1 / 2} \neq 0$.

We consider the generic option that $m_{3 / 2} \gg m_{1 / 2}$, in which there are no big bang nucleosynthesis constraints on gravitino decays, and we assume that the LSP is the lightest neutralino, so there are no problems associated with the large reheating temperature discussed earlier. In this case, however, the no-scale choices $\alpha=0$ and $\beta=1$ are the only phenomenologically viable choices. For $A_{0} \propto m_{3 / 2}$ (i.e., $\alpha \neq 0)$ and $m_{3 / 2} \gg m_{1 / 2}$, renormalization-group evolution inevitably leads to tachyonic sfermion masses. Similarly, for $\beta \neq 1$, there is no value of $\tan \beta$ which minimizes the Higgs potential.

It was shown in [29] that such no-scale boundary conditions are compatible with low-energy constraints on supersymmetry if there is a grand unification group at high scales that is broken down to $\mathrm{SU}(3) \times \mathrm{SU}(2) \times \mathrm{U}(1)$ at an appropriate high-energy scale. There are three relevant parameters in such a scenario based on $\mathrm{SU}(5)$ : the scale $M_{\text {in }}$, at which the no-scale boundary conditions are applied; and the two $\mathrm{SU}(5)$ Higgs superpotential parameters $\lambda, \lambda^{\prime}$ :, $W \ni \lambda H \Sigma \bar{H}+\left(\lambda^{\prime} / 6\right) \operatorname{Tr} \Sigma^{3}$, where $H, \bar{H}$ and $\Sigma$ are $\mathbf{5}, \overline{\mathbf{5}}$ and $\mathbf{2 4}$ Higgs representations, respectively. Regions of the no-scale SU(5) parameter space that were compatible with accelerator constraints on supersymmetric particles and the cosmological LSP density were studied in [29]. Since then, the LHC has imposed additional constraints, in particular via the Higgs discovery [30] and the decay $B_{s} \rightarrow$ $\mu^{+} \mu^{-}$[31].

Figure 2 displays a portion of the no-scale SU(5) parameter space for $\lambda^{\prime}=2$ and $\lambda=-0.1$ that is compatible with these new constraints. There is a region at low $M_{\text {in }}$ that is excluded because the LSP is charged (shaded brown), a region at large $m_{1 / 2}$ and high $M_{\text {in }}$ where we do not find consistent solutions of the renormalization-group equations (RGEs) (shaded ochre), and a region at low $m_{1 / 2}$ that is excluded by $b \rightarrow s \gamma$ (shaded green). The ratio of supersymmetric Higgs vevs, $\tan \beta$, is determined dynamically, with the indicated values along the continuous black

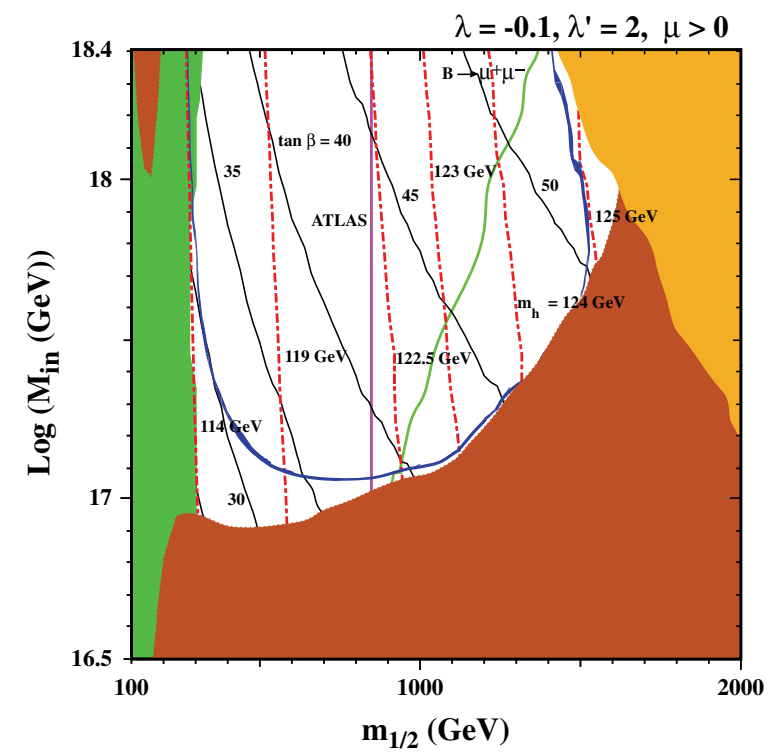

FIG. 2 (color online). The $\left(m_{1 / 2}, M_{\text {in }}\right)$ plane in the $\mathrm{SU}(5)$ noscale model, with $m_{0}=A_{0}=B_{0}=0$ at $M_{\text {in }}$, for $\lambda^{\prime}=2$ and $\lambda=-0.1$. The brown regions are excluded because the LSP is charged, the green regions are excluded by $b \rightarrow s \gamma$, the renormalization-group equations are unstable in the ochre region. The relic cold dark matter density lies within the cosmological range along the (dark blue) strips, the continuous (purple) line is the ATLAS 95\% CL limit on supersymmetric particles, the dashdotted (red) lines are contours of $m_{H}$, as calculated using FEYNHigGs [32], and the solid (green) line marks the 95\% CL upper limit on $\operatorname{BR}\left(B_{s} \rightarrow \mu^{+} \mu^{-}\right)$. The continuous (black) lines are contours of $\tan \beta$.

contours. Values of $m_{H}$ calculated with FeynHigGs [32] are shown as dash-dotted (red) contours. The area with $m_{1 / 2} \lesssim 840 \mathrm{GeV}$ (purple solid line) is excluded by ATLAS searches for supersymmetric particles at the LHC [33]. There are (dark blue) strips of parameter space extending up to $\left(m_{1 / 2}, M_{\text {in }}\right) \sim\left(1300,10^{17.5}\right) \mathrm{GeV}$ and with $m_{1 / 2} \sim$ $1500 \mathrm{GeV}$ and $M_{\text {in }} \sim 10^{18} \mathrm{GeV}$ where the relic cold dark matter density is consistent with cosmology and $m_{H} \sim 125 \mathrm{GeV}$. In view of the estimated theoretical uncertainty of $\sim 3 \mathrm{GeV}$ in the FeYNHIGGS calculation, all the portions of these dark matter strips with $m_{1 / 2} \gtrsim$ $1000 \mathrm{GeV}$ may be consistent with the LHC measurement of $m_{H}$. This region is also compatible with the experimental measurement of $B_{s} \rightarrow \mu^{+} \mu^{-}$: the diagonal (green) solid line marks the 95\% CL upper limit on this decay, including theoretical uncertainties [31]. Regions with a suitable $m_{H}$ that satisfy the other constraints can also be found for somewhat smaller values of $\lambda^{\prime}$ and $|\lambda|$.

An alternative to the pure no-scale approach taken above is the possibility that the terms in the Kähler potential corresponding to the Standard Model fields are contained either only partly within the no-scale logarithm (12) or outside it entirely via minimal kinetic terms $K \ni\left|y_{\mathrm{SM}}\right|^{2}$. For example, in the latter case, and assuming a superpotential of the form (13), the universal soft scalar mass term is now 
$m_{0}=r=m_{3 / 2}$ while the relations for $A_{0}$ and $B_{0}$ are still given by (14). It is interesting to note that for the case $\alpha=\beta=0$, one recovers the pure gravity-mediated models discussed in $[34,35]$. Viable phenomenological models can be constructed with universality input above the GUT scale [34] as in the models discussed above, or with GUT-scale universality at relatively low $\tan \beta \simeq 2$ [35]. Fixing $\tan \beta$ while satisfying the supergravity boundary conditions for $A_{0}$ and $B_{0}$ can be achieved with the addition of a GiudiceMasiero term in the Kähler potential [36].

Our analysis demonstrates that no-scale supergravity provides a suitable framework for addressing many problems in cosmology and particle physics, including inflation, leptogenesis, neutrino masses and dark matter, as well as the LHC measurement of $m_{H}$ and limits on supersymmetric particles. Concerning future experimental tests, we note that $\mathrm{CMB}$ experiments may probe the predictions of
Starobinsky-like inflationary models such as ours and that higher-energy LHC running will be sensitive to $m_{1 / 2} \lesssim 1500 \mathrm{GeV}$, the range suggested in the sample models displayed in Fig. 2. Thus, there are both cosmological and collider prospects for exploring the ideas presented here.

We thank Djuna Croon, T. Gherghetta, J. Evans, Thomas Hahn, Sven Heinemeyer, Nick Mavromatos, and Georg Weiglein for discussions. The work of J. E. was supported in part by the London Centre for Terauniverse Studies (LCTS), using funding from the European Research Council via the Advanced Investigator Grant No. 267352. The work of D. V. N. was supported in part by the DOE Grant No. DE-FG0395-Er-40917. The work of K. A. O. was supported in part by the DOE Grant No. DE-FG02-94ER-40823 at the University of Minnesota.
[1] P. Minkowski, Phys. Lett. 67B, 421 (1977); M. Gell-Mann, P. Ramond, and R. Slansky, in Supergravity, edited by D. Freedman and P. Van Nieuwenhuizen (North-Holland, Amsterdam, 1979), pp. 315-321; T. Yanagida, in Proceedings of the Workshop on the Unified Theory and the Baryon Number of the Universe, edited by O. Sawada and S. Sugamoto (KEK, Tsukuba, 1979); R. N. Mohapatra and G. Senjanovic, Phys. Rev. Lett. 44, 912 (1980).

[2] J. Wess and B. Zumino, Phys. Lett. 49B, 52 (1974); Nucl. Phys. B70, 39 (1974).

[3] A. D. Sakharov, Pis'ma Zh. Eksp. Teor. Fiz. 5, 32 (1967) [JETP Lett. 5, 24 (1967)]; , Usp. Fiz. Nauk 161, 61 (1991) [Sov. Phys. Usp. 34, 392 (1991)].

[4] F. Zwicky, Helv. Phys. Acta 6, 110 (1933); Astrophys. J. 86, 217 (1937).

[5] A. H. Guth, Phys. Rev. D 23, 347 (1981).

[6] M. Fukugita and T. Yanagida, Phys. Lett. B 174, 45 (1986).

[7] H. Goldberg, Phys. Rev. Lett. 50, 1419 (1983).

[8] J. Ellis, J. Hagelin, D. Nanopoulos, K. Olive, and M. Srednicki, Nucl. Phys. B238, 453 (1984).

[9] H. Murayama, H. Suzuki, T. Yanagida, and J. 'i. Yokoyama, Phys. Rev. Lett. 70, 1912 (1993); J. R. Ellis, M. Raidal, and T. Yanagida, Phys. Lett. B 581, 9 (2004).

[10] J. R. Ellis, K. Enqvist, D. V. Nanopoulos, K. A. Olive, and M. Srednicki, Phys. Lett. 152B, 175 (1985); 156B, 452(E) (1985); A. S. Goncharov and A. D. Linde, Classical Quantum Gravity 1, L75 (1984).

[11] J. Ellis, D. V. Nanopoulos, and K. A. Olive, Phys. Rev. Lett. 111, 111301 (2013).

[12] J. Ellis, D. V. Nanopoulos, and K. A. Olive, J. Cosmol. Astropart. Phys. 10 (2013) 009.

[13] For some representatives of other post-Planck supergravity approaches, see K. Nakayama, F. Takahashi, and T. T. Yanagida, J. Cosmol. Astropart. Phys. 08 (2013) 038; R. Kallosh and A. Linde, ibid. 06 (2013) 028; W. Buchmuller,
V. Domcke, and K. Kamada, Phys. Lett. B 726, 467 (2013); F. Farakos, A. Kehagias, and A. Riotto, Nucl. Phys. B876, 187 (2013); S. Ferrara, R. Kallosh, A. Linde, and M. Porrati, arXiv:1307.7696; J. Ellis and N.E. Mavromatos, arXiv:1308.1906.

[14] E. Witten, Phys. Lett. 155B, 151 (1985).

[15] A. A. Starobinsky, Phys. Lett. 91B, 99 (1980); V. F. Mukhanov and G. V. Chibisov, Pis'ma Zh. Eksp. Teor. Fiz. 33, 549 (1981) [JETP Lett. 33, 532 (1981)]; A. A. Starobinsky, Sov. Astron. Lett. 9, 302 (1983).

[16] P. A. R. Ade et al. (Planck Collaboration), arXiv:1303.5082.

[17] D. Z. Freedman, P. van Nieuwenhuizen, and S. Ferrara, Phys. Rev. D 13, 3214 (1976); S. Deser and B. Zumino, Phys. Lett. 62B, 335 (1976).

[18] E. Cremmer, S. Ferrara, C. Kounnas, and D. V. Nanopoulos, Phys. Lett. 133B, 61 (1983); J. R. Ellis, C. Kounnas, and D. V. Nanopoulos, Nucl. Phys. B247, 373 (1984).

[19] For an earlier example without the connection to cosmology, see S. Cecotti, Phys. Lett. B 190, 86 (1987); S. Ferrara, R. Kallosh, and A. Van Proeyen, arXiv:1309.4052.

[20] J. R. Ellis, C. Kounnas, and D. V. Nanopoulos, Phys. Lett. 143B, 410 (1984).

[21] R. Kallosh and A. Linde, J. Cosmol. Astropart. Phys. 06 (2013) 028.

[22] J. R. Ellis, A. B. Lahanas, D. V. Nanopoulos, and K. Tamvakis, Phys. Lett. 134B, 429 (1984).

[23] For a previous no-scale sneutrino inflaton model, see $\mathrm{S}$. Antusch, M. Bastero-Gil, K. Dutta, S. F. King, and P. M. Kostka, J. Cosmol. Astropart. Phys. 01 (2009) 040.

[24] D. Croon, J. Ellis, and N. E. Mavromatos, Phys. Lett. B 724, 165 (2013).

[25] J. R. Ellis, J. E. Kim, and D. V. Nanopoulos, Phys. Lett. 145B, 181 (1984); J. R. Ellis, D. V. Nanopoulos, K. A. Olive, and S. J. Rey, Astropart. Phys. 4, 371 (1996); M. Bolz, A. Brandenburg, and W. Buchmuller, Nucl. Phys. 
B606, 518 (2001);B790, 336(E) (2008); R. H. Cyburt, J. Ellis, B. D. Fields, and K. A. Olive, Phys. Rev. D 67, 103521 (2003); F. D. Steffen, J. Cosmol. Astropart. Phys. 09 (2006) 001; M. Kawasaki, K. Kohri, T Moroi, and A.Yotsuyanagi, Phys. Rev. D 78, 065011 (2008).

[26] For a recent discussion of the gravitino and moduli problems in strongly stabilized theories, see J. L. Evans, M. A. G. Garcia, and K. A. Olive, arXiv:1311.0052.

[27] I. Antoniadis, J. R. Ellis, J. S. Hagelin, and D. V. Nanopoulos, Phys. Lett. B 194, 231 (1987).

[28] J. R. Ellis, D. V. Nanopoulos, and K. A. Olive, Phys. Lett. B 300, 121 (1993).

[29] J. Ellis, A. Mustafayev, and K. A. Olive, Eur. Phys. J. C 69 , 219 (2010).

[30] G. Aad et al. (ATLAS Collaboration), Phys. Lett. B 716, 1 (2012); S. Chatrchyan et al. (CMS Collaboration), ibid. 716, 30 (2012).

[31] S. Chatrchyan et al. (CMS Collaboration), Phys. Rev. Lett. 111, 101804 (2013); R. Aaij et al. (LHCb Collaboration), ibid. 111, 101805 (2013); (LHCb and CMS Collaborations), Reports No. LHCb-CONF-2013- 012 and CMS PAS BPH-13-007.

[32] For documentation of the FeYnHigGs code, see T. Hahn, S. Heinemeyer, W. Hollik, H. Rzehak, and G. Weiglein, Comput. Phys. Commun. 180, 1426 (2009) and http:// www.feynhiggs.de/.

[33] ATLAS Collaboration, https://twiki.cern.ch/twiki/bin/ view/AtlasPublic/CombinedSummaryPlots\#SusyMSUGRA Summary.

[34] E. Dudas, A. Linde, Y. Mambrini, A. Mustafayev, and K. A. Olive, Eur. Phys. J. C 73, 2268 (2013).

[35] J. L. Evans, M. Ibe, K. A. Olive, and T. T. Yanagida, Eur. Phys. J. C 73, 2468 (2013).

[36] G. F. Giudice and A. Masiero, Phys. Lett. B 206, 480 (1988); E. Dudas, Y. Mambrini, A. Mustafayev, and K. A. Olive, Eur. Phys. J. C 72, 2138 (2012);73, 2430(E) (2013). 